\title{
Effect of loading wedge length on the propagation of stress pulse in Hopkinson bar apparatus
}

\author{
Chunhuan Guo ${ }^{1}$, Zeming Wang ${ }^{1}$, Xinhui Shen ${ }^{2}$, Guangping Zou ${ }^{2}$, Fengchun Jiang ${ }^{1 *}$ \\ ${ }^{1}$ Key Laboratory of Superlight Materials and Surface Technology, Ministry of Education, \\ College of Materials Science \& Chemical Engineering, Harbin Engineering University, Harbin, 150001, P. R. China \\ ${ }^{2}$ College of Aerospace and Civil Engineering, Harbin Engineering University, Harbin, 150001, P. R. China
}

Received 11 September 2014, received in revised form 8 December 2014, accepted 9 December 2014

\begin{abstract}
In order to study the effect of the loading wedge length on the propagation behavior of stress pulse in a Hopkinson bar loaded fracture test, non-uniform loading bars with different wedge lengths of $0,3,15.5$ and $40 \mathrm{~mm}$ have been machined from a certain rod. The Hopkinson bar loading experiment is performed on one-bar/three-point bend fracture loading system without the bending specimen to avoid the effect of specimen configuration on the reflected pulse. The results indicate that the high-frequency oscillations of the reflected pulse are enhanced and the rise time of the reflected pulse increased with increasing wedge length, i.e. the dispersion effect becomes seriously with increasing wedge length. As a consequence, the oscillations in the load-time curves determined using the incident and reflected pulses are more severe for the wedge with larger length. The loading-point displacements calculated are identical when the wedge lengths are 0 and $3 \mathrm{~mm}$. Therefore, to decrease the effect of wedge length on stress pulse propagation and to make the loading interface linear contact with a specimen, the wedge length should be designed as small as possible.
\end{abstract}

K e y w or d s: Hopkinson pressure bar, wedge-shaped, non-uniform, stress pulse

\section{Introduction}

Hopkinson pressure bar experimental techniques have been extensively employed to investigate the mechanical behavior of materials under high strain rate, such as dynamic mechanical response under tensile, compressive and torsion stress state [1]. However, the Hopkinson bar loading experimental technique that can be used to study various dynamic fracture properties of materials under a stress-intensity factor rate $\sim 10^{6} \mathrm{MPa} \mathrm{m} \mathrm{m}^{1 / 2} \mathrm{~s}^{-1}$ are still being developed [2-8]. The one-bar (one incident loading bar)/three-point bending (3PB) impact setup [9-16] is currently the most popular setup used to determine the dynamic fracture toughness of materials under stress wave loading, since this loading configuration has some advantages: (1) the loading rate acquired in such one-bar/3PB loaded fracture test is higher than that in Charpy impact test [17-26]; (2) the dynamic response of fracture specimen can be determined via strain signals recorded by the strain gauges mounted on the loading bar; and (3) a relatively large fracture specimen can be used for measuring the valid plane strain fracture toughness $[1,27-28]$. In such a onebar/3PB fracture test, in order to enable the loading bar to remain linearly in contact with the $3 \mathrm{~PB}$ fracture specimen throughout the dynamic fracture test, the loading bar is usually machined to wedge-shaped impactor, rather than a flattened end impactor, which is similar to quasi-static loading configuration [29], thus, the cross-section of the loading bar is not uniform throughout the bar. It is well known that in the Hopkinson bar loaded fracture test, the incident pulse is generated by shooting the striker bar from a gasgun and the pulse propagates along the loading bar towards fracture toughness specimen, when it reaches the interface between the end of the loading bar and fracture specimen, part of the incident pulse is reflected back into the loading bar and remainder is transmitted through the specimen to load the bending spe-

*Corresponding author: tel.: +86 451 82569890; e-mail address: fengchunjiang@hrbeu.edu.cn 
cimen to fracture. As a matter of fact, part of the incident pulse starts to reflect back early due to the wedge-shaped end of the loading bar. Before the incident pulse reaches the interface between the end of the loading bar and the 3PB fracture specimen, i.e., when the incident pulse just encounters non-uniform section of loading bar, the incident pulse reflection occurs, implying that some of the pulses is reflected from the non-uniform wedge-shaped section, rather than from the interface. Therefore, apart from the effect of the incident pulse that is related to the length, velocity of striker bar and pulse-shaping; the amplitude, duration and characteristics of the reflected pulse are affected by the wedge-shaped end of the loading bar. This is because in the one-bar/3PB fracture test, the incident and reflected pulses are only two strain signals recorded by the strain gauges mounted at the midpoint of the loading bar $[10,13,14,16]$. Using these two stress pulses, the dynamic load and loadingpoint displacement of the fracture specimen are determined and employed for calculating the dynamic stress intensity factor [5]. Combined with the crack initiating time of fracture specimen detected using an adequate method, the dynamic fracture toughness is then measured using above-mentioned procedure $[7-8$, 14]. It is clear that the accuracy of the measurement of dynamic fracture toughness is influenced by the dynamic stress intensity factor determined by two global mechanic parameters: dynamic load and displacement. These two necessary parameters are directly dependent upon the incident and reflected pulses. As a consequence, the configuration of loading bar end has a significant effect on the reflected pulse characteristics, dynamic load, displacement and stress intensity factor and dynamic fracture toughness as well. Therefore, the study associated with the effect of the loading bar end configuration on stress wave propagation behavior is a critical issue and attracted numerous researchers who performed such studies using the experimental investigation $[12,31-32,36,38-39]$, numerical simulation [11], or theoretical analysis [33-35, 37]. For example, Singh and Parameswaran [31] studied the stress-pulse dispersion caused by the rounded end of incident bar throughout experiments, and their results indicated that the wave dispersion was related to the length of the rounded end and the longitudinal pulse velocity in the elastic bar. Bacon et al. [11, 38-39] analyzed the effect of non-uniform end of incident bar on pulse distortion using both numerical simulation and experimental method, and they concluded that the stress pulse distortion was influenced by the rise time of the incident pulse and the length of the non-uniform section of incident bar. The wave dispersion generated by the non-uniform end section of the incident bar could be minimized by an incident pulse with a relatively long rise time. Zou and $\mathrm{Qu}$ [32] used numerical simulation method to invest- igate the effect of the shaped end of the incident bar on the reflected pulse and found that the dispersion of the stress pulse could be controlled via changing the length, angle, and diameter of the incident bar end. Goldsmith et al. [35, 37] experimentally studied the behavior of the elastic waves in truncated 2024 aluminum cones with different apex angles. Their results demonstrated that the truncated cone increased the peak stress at the truncated section and the increment was dependent upon both the cone angle and the length of the end section. Landon et al. [36] investigated the propagation of a compressive wave along a coned bar. Their results revealed that there was a negative tail to the wave. Ruiz et al.'s results [12] demonstrated that the rise time of the reflected pulse was significantly influenced by the alteration of wedge configuration. Suh [33] investigated the propagation behavior of compressive pulse when it propagated in a truncated cones bar against a rigid wall, and confirmed that high-frequency oscillations occurred at the end of reflected pulse, the amplitude of reflected pulse was found to decrease with increasing cross-sectional diameter of the cone. The propagation behavior of the elastic wave on the bars with various cross-sections such as round $[11,31,38$, $39]$, conic [34-37] and wedge $[12,32,33]$ has been investigated numerically and experimentally until now. Since the conclusions drawn from the previous studies seem to be ambiguous, it is therefore essential to further study the propagation behavior of the stress wave in the loading bar with non-uniform section and its effect on the determination of dynamic loading, displacement and dynamic stress intensity factor. Regardless of one-bar/3PB, two-bar/3PB [40] and threebar/3PB [41], the reflected pulse is influenced by the wave reflection in the non-uniform section of the loading bar end, while in different Hopkinson bar loading configurations, the methods to determine load and displacement are different. In two-bar/3PB setup [1], the dynamic load and displacement are determined independently using transmitted and reflected pulses; however, these two global mechanical parameters are calculated using both the incident and reflected pulses in one-bar/3PB loading system. Accordingly, the effect of the non-uniform section of the loading bar on the dynamic fracture parameters varies in different loading configurations.

It has been well-known that the non-uniformity of loading bar influences the reflected pulse characteristics, and in return influences the dynamic load and displacement, stress intensity factor, and finally causes a relatively large error in dynamic fracture toughness measurement. Therefore, in order to design and fabricate the optimized configurations of the wedge-shaped loading bar for enhancing the accuracy of dynamic fracture toughness in the one-bar/3PB fracture test, the propagation behavior of the stress pulse on the 
(a)

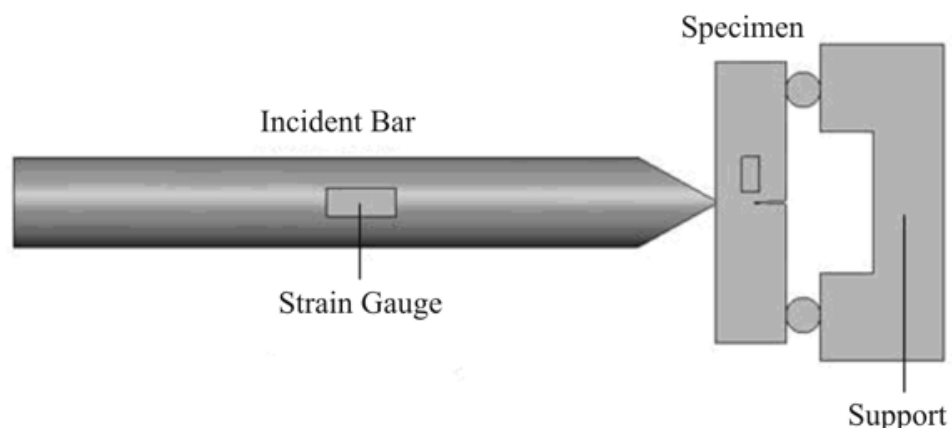

(b)
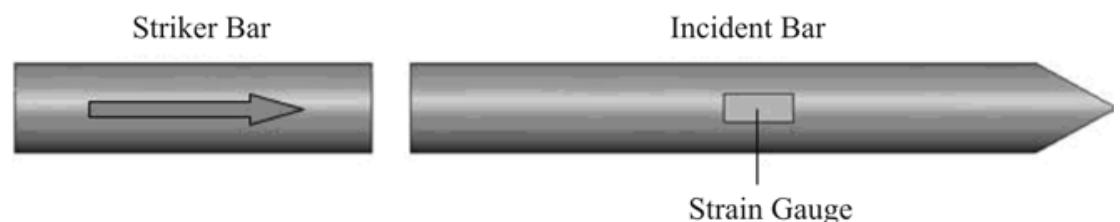

Fig. 1. The tested apparatus of Hopkinson bar: (a) The dynamic fracture tested apparatus of a one-bar/3PB experimental setup; (b) The Hopkinson bar without 3PB fracture specimen.

wedge-shaped loading bar needs to be investigated experimentally.

\section{Experimental and numerical simulation details}

To eliminate the influence of the stress pulse reflected from the interface between loading point and the impact face of the 3PB specimen when analyzing the effect of the wedge-shaped end of the loading bar on stress propagation behavior on the bar, dynamic impact tests were performed on a one-bar $/ 3 \mathrm{~PB}$ loading system without the fracture specimen, such that only the effect of the stress wave reflected from the non-uniform section of the wedge-shaped bar can be considered as a key influence factor in this work. As can be seen in Fig. 1, a modified Hopkinson pressure bar loaded dynamic fracture experimental apparatus mainly consists of the striker bar, wedge-shaped loading bar, support and data acquisition system. A thin grease foil is placed on the impact surface of the loading bar as a pulse-shaper to minimize the highfrequency oscillations in the stress pulses.

In one-bar $/ 3 \mathrm{~PB}$ fracture test, the incident $\varepsilon_{\mathrm{I}}(t)$, reflected $\varepsilon_{\mathrm{R}}(t)$ pulses are recorded by a pair of strain gauges mounted at the midpoint of the loading bar, and the dynamic load and loading-point displacement are determined using these two stress pulses in terms of one-dimensional stress wave theory as follows [30]:

$$
P(t)=E A\left[\varepsilon_{\mathrm{I}}(t)+\varepsilon_{\mathrm{R}}(t)\right],
$$

$$
\delta(t)=\int_{0}^{t} c\left[\varepsilon_{\mathrm{I}}(t)-\varepsilon_{\mathrm{R}}(t)\right] \mathrm{d} t,
$$

where $E$ is the Young's modulus, $A$ is the crosssectional area of a loading bar, $c$ is the longitudinal pulse velocity. It is obvious that the accuracy of the dynamic load and loading point displacement are directly related to the incident and reflected pulses. The amplitude, duration and characteristics of the incident pulse are mainly dependent upon the length, velocity of the striker bar, and pulse shaping, while the reflected pulse is related to not only above factors that affect the characteristics of the incident pulse but also the geometry of loading bar end. As aforementioned, the wedge shape of the loading bar has an evident effect on propagation behavior of the reflected pulse. If the effect of wedge length on the reflect pulse is neglected, a relatively large error in the determination of the dynamic load and loading point displacement and the dynamic fracture toughness will be caused.

In order to investigate the effect of wedge length on the propagation behavior of the reflected pulse, the dimension of the striker bar is fixed to be $\varnothing 14.5 \mathrm{~mm} \times 290 \mathrm{~mm}$, the diameter of the loading bar is also $\varnothing 14.5 \mathrm{~mm}$, while the lengths of wedge are machined to be $0,3,15.5$ and $40 \mathrm{~mm}$ from the same rod with $806 \mathrm{~mm}$ length, (Fig. 2). To eliminate other influenced factors created in the fabrication of the wedgeshaped bars with different wedge length and the installation of the strain gauges on a newly-machined bar, we fabricated the loading bar with various wedge lengths from the same high strength steel rod. Firstly, the experiment was performed using the loading bar 


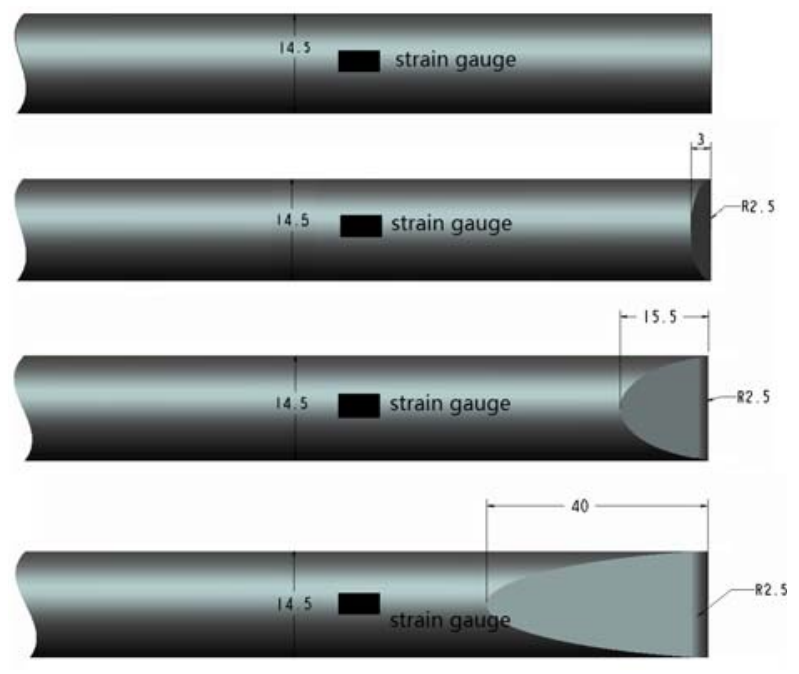

Fig. 2. Schematic diagram of the four wedge lengths used in this work: (a) $0 \mathrm{~mm}$; (b) $3 \mathrm{~mm}$; (c) $15.5 \mathrm{~mm}$; and (d) $40 \mathrm{~mm}$.

with $0 \mathrm{~mm}$ wedge (plane end), and then using the bar with $3 \mathrm{~mm}$ wedge that was directly machined from the used loading bar with $0 \mathrm{~mm}$ wedge. Similarly, the rest of experiments were conducted using the bar with the wedge lengths of 15.5 and $40 \mathrm{~mm}$. For all experiments, the incident and reflected pulses were obtained by a pair of strain gauges that are glued to the middle place of loading bar with a distance of $403 \mathrm{~mm}$ from the wedge end, so that the total length of the loading bar and the location of the strain gauges on the bar are all fixed for the four wedge lengths. The variations of the reflected pulse are therefore considered to be a result created by the lengths of the wedge-shaped end of the loading bar.

\section{Results and analysis}

\subsection{Experimentally recorded pulses}

Both incident and reflected pulses recorded from the tests performed using four loading bars are shown in Fig. 3. It is noted that the amplitude, duration and high-frequency oscillations of the incident pulse obtained experimentally are almost identical for the four loading bars with different wedge lengths (Fig. 3a), since the characteristics of the incident pulse are only dependent upon the velocity and length of the striker, and pulse shaping, as pointed out previously. However, the features of the reflected pulses are different, particularly in the initial time of reflected pulse. To clearly show the alteration of the reflected pulse obtained from the loading bars with different wedge lengths, the initial period of the reflected pulses is replotted as a function of time on a relatively large
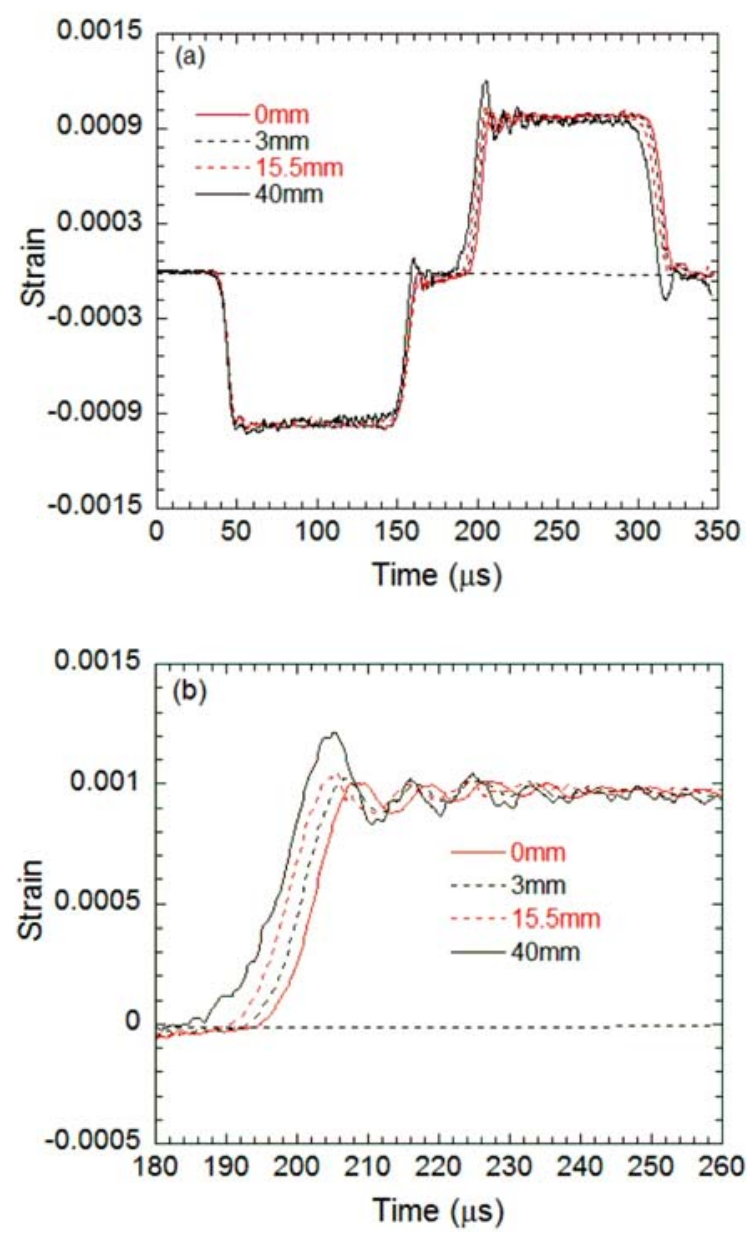

Fig. 3. The original incident and reflected pulses obtained from the loading bars with four wedge lengths. Here the stress pulses are recorded by one pair of strain gauges mounted at the fixed location on the loading bar: (a) the original stress pulses; (b) initial portion of the reflected pulse a large scale in both $X$ and $Y$ axis.

scale in $X$ and $Y$ axis, as shown in Fig. 3b. It can be found the rise time of the reflected pulse increases with increasing wedge length. The rise times of the reflected pulse can be directly determined in terms of the reflected pulses as shown in Fig. 3b, detailed measurement results for the four wedge lengths are given later in section 4 of this work. Additionally, from the comparison between the high-frequency oscillations in the incident and reflected pulses, it can be found that oscillation amplitude of the reflected pulse is greater than that of a corresponding incident pulse. It is well known that the incident and reflected pulses always have some high-frequency oscillations using loading bar without non-uniform section, which is called dispersion effect, see the stress wave of the wedge length $\sim 0 \mathrm{~mm}$ in Fig. 3a, while the dispersion effect increases with increasing wedge length. For example, in the case of the wedge length of $40 \mathrm{~mm}$, the oscilla- 
tion amplitude of the reflected pulse is nearly twice as that of the incident pulse. The bigger the length of the wedge is, the larger the high-frequency oscillations and the rise time of the reflected pulse are. Obviously, the difference in wedge lengths is for such variations in rise time and high-frequency oscillation amplitude of the reflected pulse. It should be recognized that altering the reflected pulse affected the dynamic load and loading-point displacement determined by Eqs. (1) and (2); since the incident pulses from the four loading bars are identical, the variations of the dynamic load and loading-point displacement are mainly dependent upon the behavior of the reflected pulse.

As can be seen in Fig. 3a, the experimental results indicate that the initial oscillation amplitude of the reflected pulse is higher than that of the incident pulse. Generally, the high-frequency oscillations in the incident pulse are reflected in the reflected pulse [1], i.e. the amplitude of the oscillations in the reflected pulses is dependent upon the oscillations in the incident pulses. However, in the wedge-shaped loading bar, it is also related to the wedge lengths. Goldsmith et al. [35, 37] used the initial pulse to predict the surface response in the truncated 2024 aluminum cones with different apex angles followed by the one-dimensional equation of elastic wave propagation in a cone of infinite length. Their results indicated that the peak stress increased with increasing cone angle and length of the end section, and reasonable agreement was found between the experimental data and those of calculations based on the analysis. According to the results of Goldsmith et al. $[35,37]$, it can be concluded that the amplitude of the reflected pulse increases with increasing wedge length. Therefore, the initial oscillation amplitude of the reflected pulse is higher than that of the incident pulse.

\subsection{Effect of wedge length on the rise time of reflected pulse}

As can be seen in Fig. 3, the interval time between the pulse beginning point of incident and reflected pulses for four wedge lengths are $\sim 155.4, \sim 154.8$, $\sim 152.6, \sim 150.8 \mu \mathrm{s}$, respectively. And according to one-dimensional stress theory, it is well-known that the longitudinal stress wave is calculated using the equation $C=\sqrt{E / \rho}=5189 \mathrm{~m} \mathrm{~s}^{-1}(E=210 \mathrm{GPa}$, $\left.\rho=7800 \mathrm{~kg} \mathrm{~m}^{-3}\right)$ in loading bar made of steel material. Therefore, from above data, it can be computed the position where the incident pulse begins to reflect in the non-uniform section (listed in Table 1) [42]. The results are listed in Table 1 . Table 1 shows that the reflected position increases with increasing wedge length.

It has been demonstrated [1] that the rise time of the reflected pulse is a key parameter, which plays an important role in stress state equilibrium in two-
Ta b le 1. Reflected position from the apex of wedge

Wedge length $(\mathrm{mm})$

$\begin{array}{llll}0 & 3 & 15.5 & 40\end{array}$

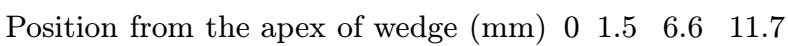

bar/3PB Hopkinson bar fracture test, so does in onebar $/ 3 \mathrm{~PB}$ fracture test when determining the dynamic load and loading point displacement. Once the incident pulses are given, the rise time, amplitude and duration of the reflected pulses are the key factors that affect the dynamic fracture parameters, and it is therefore, essential to analyze the effect of the wedge length on the rise time of the reflected pulse and on the difference between the rise times of the incident and reflected pulses. The rise times of the reflected pulses for the four wedge lengths are calculated based on the results given by experimental data (Fig. 3b). Similarly, the rise time of the incident pulses can be determined, thus the difference between the rise times of the reflected and incident pulses, $\Delta t_{\mathrm{u}}$, is calculated as

$$
\Delta t_{\mathrm{u}}=t_{\mathrm{ru}}-t_{\mathrm{iu}}
$$

where $t_{\mathrm{ru}}$ and $t_{\mathrm{iu}}$ are the rise times of reflected and incident pulses, respectively, and are determined from the experimental results. Figure 4 shows the variation of the difference of the rise time as a function of wedge length, it is noted that except for the case that the loading bar end is plane (without wedge), the variety of determined rise time of the reflected and incident pulses is greater than that by the numerical simulation, while both the variations demonstrated that the rise time difference increases with increasing wedge length, implying that the influence of the wedge length on the dynamic load and loading-point displacement determined via Eqs. (1) and (2) increases with increasing wedge length.

As can be seen in Fig. 4, for the case of the loading bar with plane end (wedge length of $0 \mathrm{~mm}$ ), the results demonstrated that the rise times of the reflected and incident pulses were almost identical, the difference between the two rise times is zero. This can be interpreted as: when the incident pulse propagates to loading bar end without wedge (it is a free end when the fracture specimen is absent), mechanical impedance suddenly drops to zero and the incident pulse is completely reflected as a tensile stress at the free end. However, for other three loading bars with different wedge lengths, mechanical impedance gradually decreases along the wedge length. When the incident pulse propagates to wedge section, a part of the incident pulse is reflected, and the remainder continues to propagate towards to the apex of the wedge. During the propagation of the rest of the incident pulse, the 


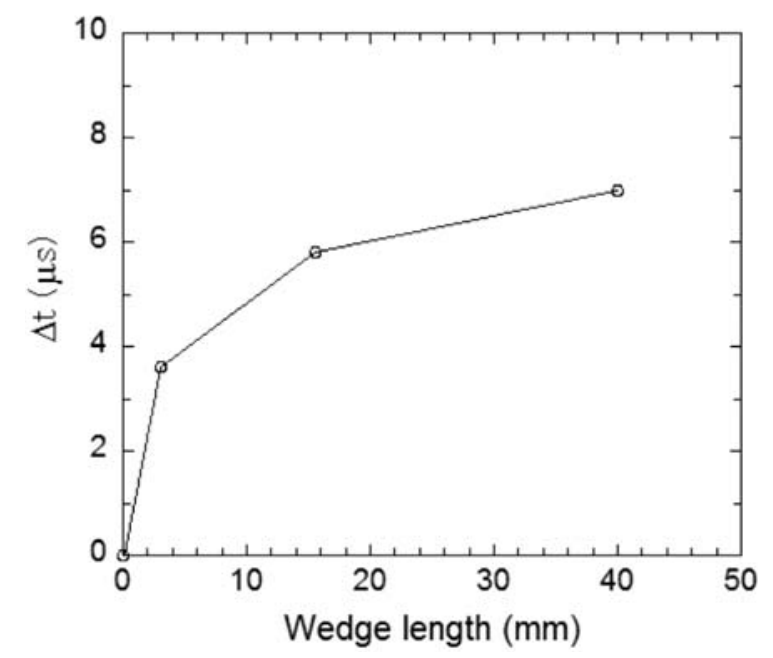

Fig. 4. Variation of the difference in rise times of the reflected and incident pulses as a function of wedge length.

wave reflection continuously occurs with the decrease of the mechanical impedance due to the alteration of the cross-section area of the wedge-shaped end. Compared to the incident pulse, the rise time of the reflected pulse is large and its slope becomes smaller. It is concluded that the longer the wedge length is, the slower the mechanical impedance changes and the bigger is the rise time of the reflected pulse.

\subsection{Effect of wedge length on load and displacement response}

To quantitatively analyze the variation of oscillations in the reflected pulse created by the wedgeshaped end of the loading bar, the parameter $\Delta$ is used to represent the variation of oscillation amplitude of the reflected pulse, which is defined as:

$$
\Delta=\frac{\varepsilon_{\mathrm{rmax}}-\varepsilon_{\mathrm{rmin}}}{\varepsilon_{\mathrm{ra}}} \times 100 \%,
$$

where $\varepsilon_{\mathrm{ra}}$ is the average amplitude that is corresponding platform of the reflected pulse, $\varepsilon_{\text {rmax }}$ and $\varepsilon_{\text {rmin }}$ are the maximum and minimum amplitudes of oscillations of the reflected pulse, respectively, which are determined from the stress pulses (Fig. 3). Figure 5 shows the variation of oscillation amplitude of the reflected pulses as a function of time for the loading bars having various wedge lengths. It is clear that the variation of oscillation amplitude of the reflected pulses determined increases with increasing wedge length.

Theoretically, the wedge section can be considered as a step bar consisting of a series of different crosssectional areas [43]. When incident compressive pulse propagates from the root of wedge (intersection with cylinder of loading bar) to its apex, it passes through

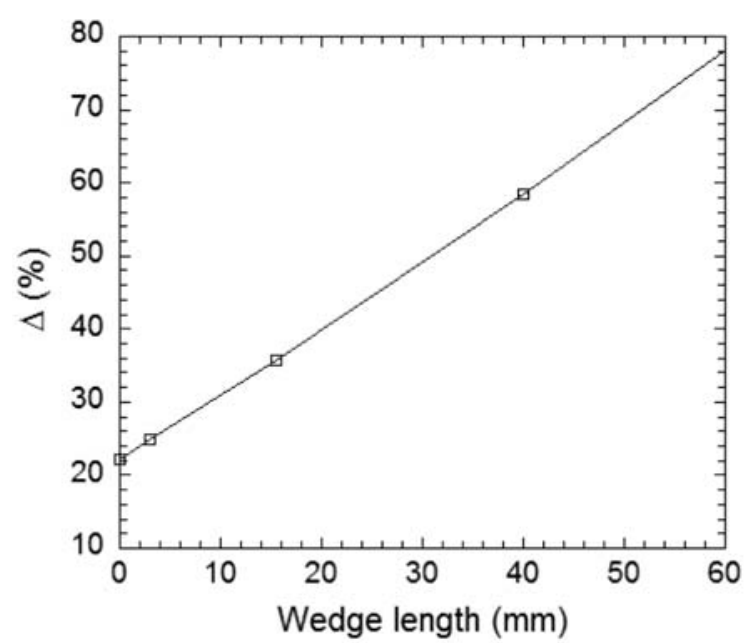

Fig. 5. Variation of oscillation amplitude of the reflected pulses as a function of wedge length.

each cross section with given areas (area of $n$-th cross section is assumed as $A_{n}$ ) and intensity of transmitted compressive pulse will increase to $\frac{2 A_{n}}{A_{n}+A_{n+1}}$ times as the former (here $A_{n+1}$ is the area of $(n+1)$-th cross section), meanwhile, a tensile pulse is reflected. Predictably, as incident compressive pulse propagates along the wedge to the apex of wedge, the amplitude of stress in compressive zone at pulse front will become larger and larger, and the amplitude of tensile pulse in tensile zone that is created following the compressive zone also becomes larger and larger [35, 37]. At the same time, some regions of the wedge section are in compressive stress state, and others are in tensile stress state. With the time passing, there is an alternation between compressive and tensile stress states in the same region. Therefore, during the propagation of the stress wave across the wedge-shaped end, the stress distribution along the wedge length is in a nonuniform state, the non-uniform stress state surely creates an evident effect on reflected pulse. Obviously, such an effect is dependent upon the wedge length.

As demonstrated previously in this work, the reflected pulse is influenced by the wedge length; the dynamic load is therefore affected by the reflected pulse, as there is a direct linear relationship between dynamic load and the reflected pulse as shown in Eq. (1). The effect of the wedge length on the dynamic load response can be found in Fig. 6, in which the variation of the applied dynamic load as a function of time is shown. Clearly, the peak load varies as a function of wedge length, and it increases with increasing wedge length, the loading bar with wedge length of $40 \mathrm{~mm}$ has a maximum load compared to others. For the case of the loading bar without wedge, theoretically speaking, there should be no oscillations in load-time re- 


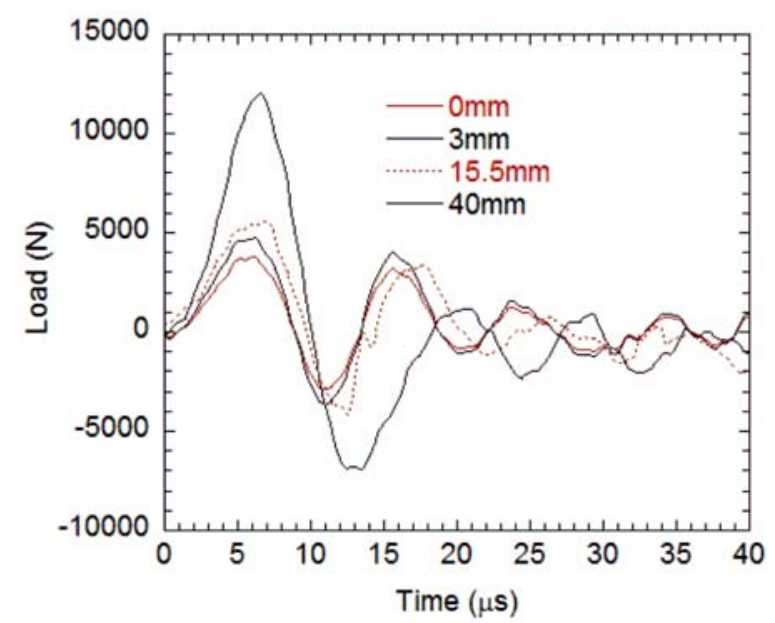

Fig. 6. The dynamic load response as a function of time for the four loading bars with various wedge lengths. Here the dynamic load is determined using the measured stress pulses via Eq. (1).

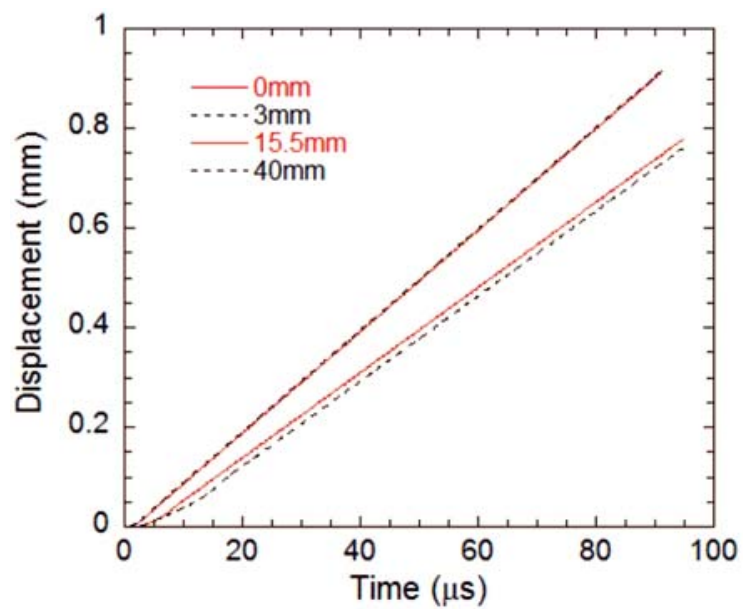

Fig. 7. Variations of the loading point displacements given by Eq. (2).

sponse curve created, while the oscillations with relatively large amplitude are still seen at the initial time of the load vs. time response, which are ProchhammerChree oscillations [8], the inherent nature of the stress wave propagation along the elastic bar even in pulseshaped Hopkinson bar test.

The loading-point displacement can be experimentally measured using the incident and reflected pulses via Eq. (2). For comparison, the loading-point displacements determined are plotted as a function of time in one graph for each wedge length in Fig. 7. Clearly, the results demonstrated that the loadingpoint displacements for the wedge lengths of 0 and $3 \mathrm{~mm}$ are identical while the displacements determined from other wedge lengths deviate from that for wedge length of $0 \mathrm{~mm}$ with increasing time. For the wedge lengths of 15.5 and $40 \mathrm{~mm}$, the loading-point displacements are both smaller compared to the wedge lengths of 0 and $3 \mathrm{~mm}$. It can be concluded that if the wedge length is machined shorter, the results will be more accurate.

\section{Conclusions}

In order to thoroughly understand the effect of wedge length of loading bar on the propagation behavior of the stress-pulse in non-uniform section (wedge), and on the dynamic load and loading-point displacement, dynamic fracture experiments without $3 \mathrm{~PB}$ fracture specimen were performed in one-bar $/ 3 \mathrm{~PB}$ fracture tests, the conclusions drawn from this work are as follows:

1. The high-frequency oscillations of the reflected pulse are enhanced intensely due to wedge shaped end of the loading bar, and the oscillation amplitude increases with increasing wedge length, which influences the dynamic responses of the load and loading-point displacement that are dependent upon the incident and reflected pulses. That is to say, the wedge length affects the stress pulse propagation, which brings about the dispersion effect on the reflected pulse;

2. The rise time of the reflected pulse is greater than that of a corresponding incident pulse for each wedge length. The rise time of the reflected pulse and difference between the rise times of the incident and reflected pulses increase with increasing wedge length;

3. The loading-point displacements for the wedge lengths of 0 and $3 \mathrm{~mm}$ are identical while a relatively large deviation in loading-point displacements is found for the wedge lengths of 14.5 and $40 \mathrm{~mm}$. Therefore, to decrease the effect of wedge length on stress pulse propagation and make the loading interface linear contact with a specimen, the wedge length should be designed as small as possible.

\section{Acknowledgements}

The authors gratefully acknowledge the financial support of this study by the National Natural Science Foundation of China (No.11172074 and No.11402060) and the Fundamental Research Funds for the Central Universities (HEUCF201403014).

\section{References}

[1] Jiang, F., Vecchio, K. S.: Applied Mechanics Reviews, 62, 2009, p. 060802 1. doi:10.1115/1.3124647

[2] Rittel, D., Maigre, H., Bui, H. D.: Scripta Metallurgica Materialia, 26, 1992, p. 1593. doi:10.1016/0956-716X(92)90262-D 
[3] Bui, H. D., Maigre, H., Rittel, D.: International Journal of Solids Structure, 29, 1992, p. 2881. doi:10.1016/0020-7683(92)90146-K

[4] Homma, H., Kanto, Y., Tanaka, K.: Journal de Physique IV, 1, 1991, p. 589. doi:10.1051/jp4:1991383

[5] Jiang, F., Rohatgi, A., Vecchio, K. S., Cheney, J. L.: International Journal of Fracture, 127, 2004, p. 147. doi:10.1023/B:FRAC.0000035058.03627.30

[6] Weerasooriya, T., Moy, P., Casem, D., Cheng, M., Chen, W.: Journal of American Ceramic Society, 89, 2006, p. 990. doi:10.1111/j.1551-2916.2005.00896.x

[7] Jiang, F., Vecchio, K. S.: The Review of Scientific Instruments, 78, 2007, p. 063903. doi:10.1063/1.2746630

[8] Jiang, F., Vecchio, K. S.: Metallurgical and Materials Transactions A, 38, 2007, p. 2896. doi:10.1007/s11661-007-9301-8

[9] Bacon, C.: Measurement of Dynamic Toughness of Brittle Materials in Flexion-Three-Point-Temperature AHIGH Using Hopkinson Bars. [Ph.D. Thesis] Bordeaux, University of Bordeaux 1993. (in French)

[10] Bacon, C., Farm, J., Lataillade, J. L.: Experimental Mechanics, 34, 1994, p. 217. doi:10.1007/BF02319758

[11] Bacon, C.: International Journal of Impact Engineering, 13, 1993, p. 527. doi:10.1016/0734-743X(93)90084-K

[12] Ruiz, C., Mines, R. A. W.: International Journal Fracture, 29, 1985, p. 101. doi:10.1007/BF00034296

[13] Popelar, C. H., Anderson, C. E. Jr., Nagy, A.: Experimental Mechanics, 40, 2000, p. 401. doi: $10.1007 / \mathrm{BF} 02326486$

[14] Rubio, L., Fernández-Sáez, J., Navarro, C.: Experimental Mechanics, 43, 2003, p. 379. doi: $10.1007 / \mathrm{BF} 02411342$

[15] Loya, J., Fernández-Sáez, J., Navarro, C.: Journal de Physique IV, 110, 2003, p. 305. doi:10.1051/jp4:20020711

[16] Loya, J. A., Fernández-Sáez, J.: International Journal of Solids Structure, 45, 2008, p. 2203. doi:10.1016/j.ijsolstr.2007.11.027

[17] Mines, R. A. W., Ruiz, C.: Journal de Physique IV, 46, 1985, p. 187. doi:10.1051/jphyscol:1985524

[18] Böhme, W., Kalthoff, J. F.: International Journal of Fracture, 20, 1982, p. R139. doi:10.1007/BF01130620

[19] Kalthoff, J. F.: International Journal of Fracture, 27, 1985, p. 277. doi: $10.1007 / \mathrm{BF} 00017973$

[20] Crouch, B. A.: Computer Structure, 48, 1993, p. 167. doi:10.1016/0045-7949(93)90468-S

[21] Rokach, I. V.: Composites and Adhesives. New York, Elsevier 2000.

[22] Marur, P. R.: Engineering Fracture Mechanics, 61, 1998, p. 369. doi:10.1016/S0013-7944(98)00064-2

[23] Marur, P. R., Simha, K. R. Y., Nair, P. S.: International Journal of Fracture, 68, 1994, p. 261. doi:10.1007/BF00013071
[24] Landrein, P., Lorriot, T., Guillaumat, L.: Engineering Fracture Mechanics, 68, 2001, p. 1631. doi:10.1016/S0013-7944(01)00062-5

[25] Lorriot, T., Martin, E., Quenisset, J. M., Rebiere, J. P.: International Journal of Fracture, 91, 1998, p. 299. doi:10.1023/A:1007583731072

[26] Jian, F., Fulian, D., Chengzhong, W.: Journal de Physique IV, 110, 2003, p. 551. doi:10.1051/jp4:20020751

[27] Böhme, W.: Impact Loading and Dynamic Behavior of Materials. Oberursel, DGM Informationsgesellschaft Verlag 1988.

[28] Vecchio, K. S., Jiang, F.: Metallurgical and Materials Transactions A, 38, 2007, p. 2655. doi:10.1007/s11661-007-9204-8

[29] Standard Test Method for Linear-Elastic Plane-Strain Fracture Toughness KIC of Metallic Materials, ASTM E399-09.

[30] Meyers, A. M.: Dynamic Behavior of Materials. New York, A Wiley-Interscience Publication 1994.

[31] Singh, R. P., Parameswaran, V.: Optical Lasers Engineering, 40, 2003, p. 289. doi:10.1016/S0143-8166(02)00089-1

[32] Zou, G., Qu, J.: Key Engineering Materials, 334-335, 2007, p. 153. doi:10.4028/www.scientific.net/KEM.334-335.153

[33] Suh, N. P.: Experimental Mechanics, 12, 1967, p. 541. doi:10.1007/BF02326331

[34] Rader, D., Mao, M.: Experimental Mechanics, 2, 1972, p. 90. doi:10.1007/BF02408444

[35] Kenner, V. H., Goldsmith, W.: Experimental Mechanics, 8, 1968, p. 442. doi:10.1007/BF02327408

[36] Landon, J. W., Quinney, H.: Proceedings of the Royal Society of London. Series A, Containing Papers of a Mathematical and Physical Character, 103, 1923, p. 622. doi:10.1098/rspa.1923.0084

[37] Lewis, J. L, Goldsmith, W., Cunningham, D. M.: Experimental Mechanics, 9, 1969, p. 313. doi:10.1007/BF02325137

[38] Bacon, C.: Journal of Applied Mechanics, 61, 1994, p. 493. doi:10.1115/1.2901480

[39] Bacon, C., Brun, A.: International Journal of Impact Engineering, 24, 2000, p. 219. doi:10.1016/S0734-743X(99)00166-9

[40] Tanaka, K., Kagatsume, T.: Bullet of JSME, 23, 1980, p. 1736. doi:10.1299/jsme1958.23.1736

[41] Yokoyama, T.: ASME Journal of Pressure Vessel Technique, 115, 1993, p. 389. doi:10.1115/1.2929546

[42] Rader, D., Mao, M.: Experimental Mechanics, 12, 1972, p. 90. doi:10.1007/BF02408444

[43] Wasley, R. J.: Stress Wave Propagation in Solids. New York, Marcel Dekker Inc. 1973. 